\title{
Apoptosis Induced by Pseudomonas aeruginosa in Antigen Presenting Cells Is Diminished by Genetic Modification with CD40 Ligand
}

\author{
STEFAN WORGALL, KATHERINE MARTUSHOVA, ANNETTE BUSCH, LEAH LANDE, AND \\ RONALD G. CRYSTAL
}

\begin{abstract}
Division of Pulmonary and Critical Care Medicine [S.W., K.M., L.L., R.G.C.], Department of Pediatrics [S.W.], Belfer Gene Therapy Core Facility [A.B., R.G.C.], and Department of Genetic Medicine [S.W., R.G.C.], Weill Medical College of Cornell University, New York, New York 10128, U.S.A.
\end{abstract}

\begin{abstract}
Persistent colonization with Pseudomonas aeruginosa (PA) is a hallmark of the lung disease associated with cystic fibrosis (CF). Based on the concept that PA is not cleared from the lung by the host response in individuals with $\mathrm{CF}$, we analyzed the capacity of PA to induce cell death in human alveolar macrophages (AM) and murine dendritic cells (DC), antigen presenting cells that play a central role in the initiation of pulmonary host defenses against pathogens, and evaluated if genetic modification can lead to protection against PA induced cell death. AM and DC were susceptible to cell death induced by the laboratory PA isolates PAO1, PAK and PA103, as well as a mucoid derivative of PAO1 and PA isolates derived from sputum of individuals with CF. Apoptosis, analyzed by TUNEL assay, was detectable in AM and DC as early as $3 \mathrm{~h}$ after infection with PA. In contrast, the same strains and doses of PA had little effect on the lung epithelial cell line A549 and primary cultures of human bronchial epithelial cells in vitro. Pretreatment of DC with the caspase inhibitors VAD-fmk and YVAD-cmk reduced PA induced cell death $(p<0.05)$. Finally, genetic modification of DC to express
\end{abstract}

\section{ABSTRACT}

CD40L using an adenovirus vector decreased the susceptibility of DC to cell death induced by PAO1 compared with DC infected with a control Ad vector $(p<0.01)$. The data demonstrate that DC and AM are susceptible to apoptosis induced by PA and that this response can be partially reversed by genetic modification with CD40L, a CD4 $+\mathrm{T}$ cell molecule that plays a central role in activating antigen presenting cells. These observations suggest a potential mechanism contributing to the persistence of PA in CF and suggest that genetic manipulation of antigen presenting cells with anti-apoptotic genes may be able to strengthen host defenses in CF. (Pediatr Res 52: 636-644, 2002)

Ad, adenovirus
AM, alveolar macrophages
CF, cystic fibrosis
DC, dendritic cells
PA, Pseudomonas aeruginosa

Pseudomonas aeruginosa (PA) can cause infections of the respiratory tract, particularly in immunocompromised patients or individuals with cystic fibrosis (1-3). Of particular interest is the interaction of PA with cells of the respiratory system, a major site for infections with PA. The mechanism by which PA can establish infection in the lung is undoubtedly complex and linked in part to the ability of the PA population to develop biofilms, and in part by the capacity of PA to interfere with the

Received September 24, 2001; accepted April 24, 2002

Correspondence: Stefan Worgall, Institute of Genetic Medicine, Weill Medical College of Cornell University, 515 East 71st Street, Suite 1000, New York, New York 10021, U.S.A.; e-mail: geneticmedicine@med.cornell.edu

These studies were supported, in part, by P01 HL51746; P01 HL59312; R01 HL 59861; M01RR00047; the Will Rogers Memorial Fund, Los Angeles, CA, U.S.A.; the Cystic Fibrosis Foundation, Bethesda, MD, U.S.A.; and GenVec, Inc., Gaithersburg MD, U.S.A. S. Worgall is supported, in part, by the Francis Families Foundation as a Parker B. Francis Fellow in Pulmonary Research.

DOI: 10.1203/01.PDR.0000032382.17567.FB host defense mechanisms arrayed against pathogens (1-5). Critical to the pulmonary defense against extracellular bacteria is the sampling and processing of the bacteria by antigen presenting cells, including alveolar macrophages (AM) and dendritic cells (DC). AM play a central role in clearing extracellular bacteria from the lung and DC interspersed in the airway epithelial cells and in the lung parenchyma are the most potent antigen presenting cells in the lung (6-9).

The focus of the present study is to analyze whether antigenpresenting cells can be protected from PA induced apoptosis by prior genetic modification. Several recent studies have shown that PA can be cytotoxic, and can induce apoptosis in a variety of cell types such as epithelial cells, macrophages and endothelial cells (10-16). Multiple virulence factors of PA, in particular the type III secretion system, have been proposed to be important for this process $(11,13,14,16)$. Induction of apoptosis in antigen presenting cells is a major virulence factor 
for enteral pathogens such as Shigella, Salmonella and E. coli (17-21), leading to impairment of the intestinal host defense system and thus contributing to the spread of the infection. The induction of apoptosis with release of cytokines in intestinal macrophages by Salmonella or Shigella is an important characteristic of the host-pathogen interaction for these bacteria $(17,19,21,22)$.

In the context that induction of apoptotic cell death by PA in antigen presenting cells might be one mechanism for this virulence in the lung environment and thus contribute to the progression of the infection, the present study extends the concept of PA induced apoptosis to evaluate AM and DC, two cell types critical to the clearance of extracellular bacteria from the lung. The data demonstrate that a variety of established laboratory as well as clinical isolates of PA are capable of inducing apoptosis in AM and DC. Importantly, we also show that at least partial protection against PA induced apoptosis of antigen presenting cells can be achieved by prior genetic modification of the antigen presenting cell with $\mathrm{CD} 40 \mathrm{~L}$, a member of the TNF superfamily normally expressed on activated CD4+ lymphocytes. CD40L acts via ligation to its receptor CD40, expressed on antigen presenting cells (23). In addition to being a critical mediator of $\mathrm{T}$ cell dependent $\mathrm{B}$ cell activation (24), CD40 ligation on DC has been shown to lead to enhanced activation and differentiation as well as to decreased tumor induced apoptosis (25). Since DC genetically modified to express $\mathrm{CD} 40 \mathrm{~L}$ and pulsed with heat inactivated PA provide enhanced protection and induction of a strong anti-PA antibody response against chronic pulmonary PA infection (26), genetic modification of antigen presenting cells to express CD40L may prove useful for novel strategies of protecting the susceptible host against PA.

\section{METHODS}

Bacteria strains. The PA strains used included the well characterized laboratory strains PAOI, PAO/NP, PAK and PA103 (provided by A. Prince, Columbia University, NY, U.S.A.), a strain isolated from the sputum of an individual with CF (AD2A provided by J. Burns, University of Washington, WA, U.S.A.), and the mucoid laboratory strain PDO300, a mucA22 derivative of PAO1 constructed by crossing a mucA22 fragment from FRD1 into the PAO1 genome by gene replacement (provided by D. Ohman, Commonwealth University of Virginia, VA, U.S.A.). PA LPS and E. coli LPS was obtained from Calbiochem, San Diego, CA, U.S.A. Bacteria were grown from frozen stocks in tryptic soy broth (Difco, Detroit, MI, U.S.A.) at $37^{\circ} \mathrm{C}$ to midlog phase, washed three times with PBS, pH 7.4 (PBS) and resuspended in infection media at the desired concentration as determined by spectrophotometry. For different inoculum doses within an individual experiment serial dilutions were done and the inoculum volume kept constant. Numbers of bacteria were confirmed by determining the colony forming units (cfu) of diluted aliquots on MacConkey agar plates (Difco). PDO300 colonies always demonstrated a mucoid phenotype. Bacteria were heat inactivated at $56^{\circ} \mathrm{C}$ for $1 \mathrm{~h}$.
Cells. Murine bone marrow-derived DC were generated from 6 to 8 wk old C57Bl/6 mice (Jackson Laboratories, Bar Harbor, ME, U.S.A.) and grown in complete RPMI 1640 medium ( $10 \%$ fetal bovine serum, $2 \mathrm{mM}$ L-glutamine, 100 $\mu \mathrm{g} / \mathrm{mL}$ streptomycin, and 100 units $/ \mathrm{mL}$ penicillin) supplemented with $10 \mathrm{ng} / \mathrm{mL}$ recombinant mouse granulocyte macrophage colony stimulating factor (GM-CSF, R\&D systems, Minneapolis, MN, U.S.A.) and $2 \mathrm{ng} / \mathrm{mL}$ recombinant mouse IL-4 (IL-4, R\&D systems) as previously described $(26,27)$.

Human alveolar macrophages were obtained by bronchoalveolar lavage of healthy volunteers (28). The lavage fluid was filtered through one layer of gauze, centrifuged $(400 \mathrm{~g}, 10 \mathrm{~min})$ and washed three times in PBS, pH 7.4. Cells were resuspended in complete RPMI 1640 medium and plated. Macrophage content (always $>90 \%$ ) was determined by Giemsa stain on cytospin preparations. Cell viability (always $>90 \%$ ) was determined by trypan blue exclusion. After $3 \mathrm{~h}$, the macrophages were washed to remove nonadherent cells.

The human respiratory epithelial cell line A549 was maintained in Dulbecco's Modified Eagle Medium (10\% fetal bovine serum, $2 \mathrm{mM}$ L-glutamine, $100 \mu \mathrm{g} / \mathrm{mL}$ streptomycin, and $100 \mathrm{units} / \mathrm{mL}$ penicillin). Primary human bronchial epithelial cells were obtained from Clonetics (Walkersville, MD, U.S.A.) and maintained in Bronchial Epithelial Basal Medium with growth factors and supplements as supplied by Clonetics.

Adenovirus vectors. $\mathrm{AdmCD} 40 \mathrm{~L}$ is a replication-deficient Ad5-based recombinant adenovirus with E1 and E3 deletions in which the mouse CD40L cDNA is under control of the cytomegalovirus immediate-early promoter and enhancer (29). The control AdNull vector is structurally similar but contains no transgene (30). Preparation and titration of the adenovirus vectors were as previously described $(31,32)$. All vectors used in this study were free of replication competent adenovirus (33).

Cytotoxicity of PA. To evaluate the cytotoxicity of PA strains on antigen presenting cells, DC and AM were plated in 96 well plates at $5 \times 10^{4} /$ well and infected with PA at doses ranging from 0.1-100 bacteria/cell in RPMI, 25 mM HEPES, $\mathrm{pH}$ 7.4. Immediately after addition of the bacteria, the plates were centrifuged at $500 \times \mathrm{g}$ for $10 \mathrm{~min}$ to synchronize the initiation of the infection. Cytotoxicity was assessed by measuring LDH release into the supernatant after $3 \mathrm{~h}$ using a colorimetric assay (Roche, Indianapolis, IN, U.S.A.) and measuring the OD at $450 \mathrm{~nm}$. Maximum LDH release was determined by addition of $1 \%$ Triton $\mathrm{X}$ to uninfected cells at the initiation of the infection. A549 and HBEC were infected in a similar fashion in their media supplemented with $25 \mathrm{mM}$ HEPES, pH 7.4.

$P A$-induced apoptosis in antigen presenting cells. To assess whether PA induces apoptosis in antigen presenting cells, DC and A549 cells were infected with PA as described above in 96 well plates for $3 \mathrm{~h}$ and apoptosis was assessed by a colorimetric assay for DNA fragmentation (R\&D Systems) as described by the manufacturer. Cells spiked with PA for $0 \mathrm{~h}$ served as a negative control, cells treated with DNAse served as a positive control. To evaluate the induction of apoptosis using a different method, DC and A549 cells were plated in coverslip dishes and infected with PA for $3 \mathrm{~h}$. The cells were then washed twice and 
continued in culture in the presence of gentamicin $(200 \mu \mathrm{g} / \mathrm{mL}$; Sigma Chemical Co., St. Louis, MO, U.S.A.) for 0 or 3 h. Cells were then fixed with $4 \%$ paraformaldehyde for $15 \mathrm{~min}$, permeabilized with $0.2 \%$ Triton X-100 for 5 min and DNA strand breaks were detected by in situ terminal deoxynucleotidyltransferase (TUNEL) assay (Promega, Madison, WI, U.S.A.) using fluorescein thiocyanate conjugated dUTP. Positive controls were generated by treating uninfected cells with DNAse. Nuclei were counterstained with propidium iodide. The number of apoptotic cells was determined by fluorescent microscopy. Apoptosis was also evaluated using binding of annexin V. DC were infected with PAO1 at $1 \mathrm{cfu} / \mathrm{cell}$ for $4 \mathrm{~h}$, washed and stained with annexin V-FITC (Pharmingen), counterstained with $2 \mu \mathrm{M}$ TO-PRO3-iodide (Molecular Probes, Eugene, OR, U.S.A.) to detect dead cells and then analyzed by flow cytometry.

Effect of capase inhibition on PA induced cellular cytotoxicity. To analyze whether PA induced cytotoxicity can be influenced by caspase inhibition, murine DC were incubated with the irreversible caspase inhibitors VAD-fmk $(40 \mu \mathrm{M}$, nonspecific inhibitor of caspase-3 and 8) or YVAD-cmk (40 $\mu \mathrm{M}$, nonspecific inhibitor of caspase-1; both from Clontech, Palo Alto, CA, U.S.A.) in RPMI for $1 \mathrm{~h}$ before addition of PAO1 (10 cfu/cell) or PA103 (1 cfu/cell) for $1 \mathrm{~h}$. PA induced cell death was then assessed by measuring LDH release in the supernatant as described above.

\section{Effect of genetic modification with CD40L}

To evaluate the effect of genetic modification with CD40L on PA induced cell death in antigen presenting cells, DC were infected with AdCD40L or Ad Null at 100 moi. After $24 \mathrm{~h}$ the cells were infected with PAO1 or PDO300 for $3 \mathrm{~h}$ and cell death was evaluated by TUNEL assay and LDH release as described above. Expression of CD40L, MHC class II and ICAM-1 (CD54) was evaluated by flow cytometry (Coulter, Miami, FL, U.S.A.) using phycoerythrin (PE)-labeled antibodies (all from PharMingen, San Diego, CA, U.S.A.). An isotypematched mouse $\mathrm{mAB}$ was used as negative control (PharMingen).

\section{Statistical Analysis}

All data are reported as mean \pm SE. Statistical evaluation was performed using the one-tailed $t$ test, and a value of $p<$ 0.05 was accepted as a significant difference.

\section{RESULTS}

$P A$ Induced cell death of antigen presenting cells. To analyze the effect of PA on antigen presenting cells, human $\mathrm{AM}$ and murine DC were infected with the laboratory PA strains PAO1, PAO/NP, PAK, and PA103, and cell death evaluated by measuring LDH release after $3 \mathrm{~h}$. All PA strains caused cytotoxicity of both AM and DC, in contrast to the lung epithelial cell line A549, where only PA103 caused cell death at this time point (Fig. 1). The effect was dose dependent and was dependent on the PA strain (PAO1 and PA103 > PAO/NP $>$ PAK).
A.

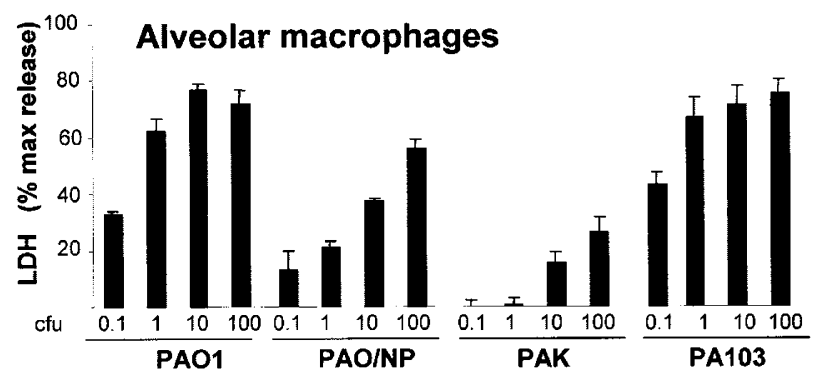

B.

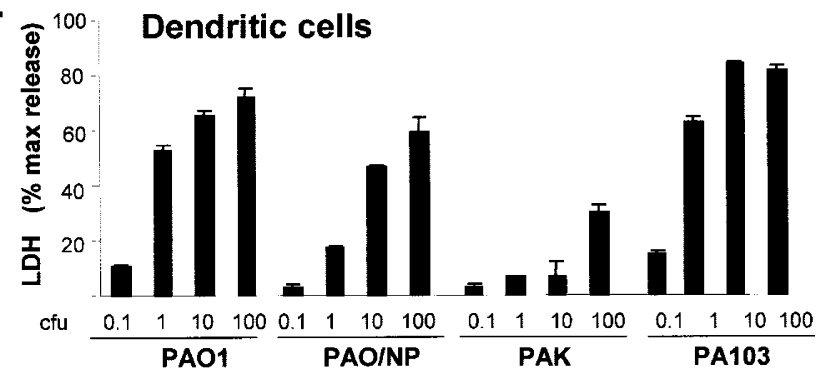

C.

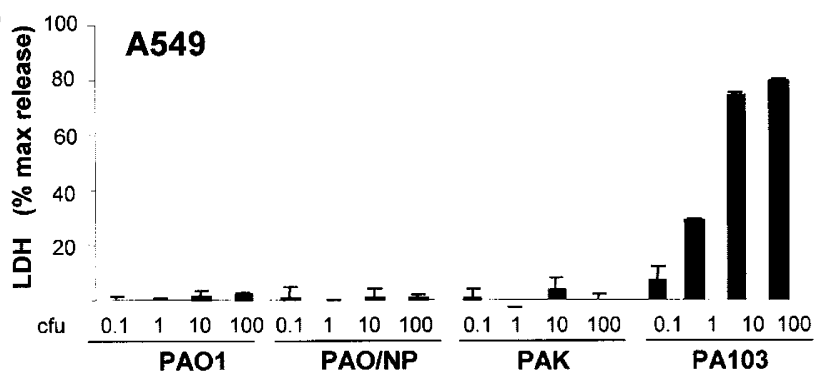

Figure 1. Cytotoxicity of antigen presenting cells induced by different laboratory and clinical strains of Pseudomonas aeruginosa. Human AM obtained by bronchoalveolar lavage from healthy volunteers. DC were derived from bone marrow of $\mathrm{C} 57 \mathrm{Bl} / 6$ mice. The $\mathrm{AM}, \mathrm{DC}$, and the human lung epithelial cell line A549 were infected with various laboratory strains of PA at doses from $0.1-100 \mathrm{cfu} / \mathrm{cell}$. Following addition of the bacteria, the plates were centrifuged to synchronize the initiation of the infection. Cytotoxicity was assessed by measuring LDH release into the supernatant using a colorimetric assay following $3 \mathrm{~h}$ infection. $(A) \mathrm{AM} ;(B)$ dendritic cells, and (C) A549 cells. Data are presented as LDH in supernatant/maximum cellular release of LDH by lysis with triton-X above the baseline LDH release for uninfected cells. Shown is a representative of three experiments. Each datapoint represents mean of triplicate wells

To analyze whether clinical PA isolates derived from individuals with cystic fibrosis or a mucoid laboratory isolate are equally able to induce cell death in DC as laboratory strains of $\mathrm{PA}$, a clinical isolate derived from an individual with $\mathrm{CF}$, AD2A, and a mucoid PA mutant PD0300 were used to infect DC. The clinical PA isolate (Fig. $2 A$ ) as well as the mucoid mutant PDO300 (Fig. 2B) induced dose-dependent cell death in DC comparable to the parental strain PAO1, suggesting that cell death in DC can be induced by various phenotypes. The clinical and mucoid strains seemed to be less cytotoxic compared with PAO1 or PA103. Similar results were obtained when other clinical isolates, derived at different stages of disease from the CF lung were evaluated (not shown). To evaluate the role of bacterial membrane components, in particular LPS, DC were exposed to $100 \mathrm{cfu} / \mathrm{mL}$ of heat inacti- 
vated PAO1 or PA103 and LPS, derived from E. coli or PA. No significant cell death was observed after 3 h (Fig. 2C), suggesting that the induction of cell death is an active process by live PA.

To evaluate whether the observed cell death was due to apoptosis, DC and A549 cells were infected with the same PA strains; apoptosis was evaluated using the TUNEL assay. Apoptotic AM and DC were detectable by TUNEL assay following infection with PAO1 after $3 \mathrm{~h}$, whereas no significant apoptosis was observed in the A549 cell infected with the same dose of PAO1 (Fig. 3). At $6 \mathrm{~h}$ following infection, apoptotic A549 cells were seen in the cells infected with the strain PA103, whereas exposure of A549 cells to PAK, PAO1 or $\mathrm{PAO} / \mathrm{NP}$ did not result in significant apoptosis (Fig. 4). In contrast, apoptosis in $\mathrm{DC}$ was detectable at $3 \mathrm{~h}$ with not only PA103, but was also observed with PAO1, PAO/NP and PAK (Fig. 4). Similar results were obtained using a colorimetric assay measuring DNA breaks (not shown). Using annexin V binding as another assay for the detection of apoptosis showed increased dead and apoptotic cells in DC infected with PAO1 (Fig. 5). Quantification showed $42.7 \pm 7.4 \%$ annexin $V$ positive cells infected with PAO1 compared with $10.0 \pm 2.8 \%$ in uninfected controls, the number of To-Pro-3-iodide positive DC was $60.7+5.0$ for infected compared with $17.8+2.2$ for uninfected cells. These results suggest that $\mathrm{AM}$ and $\mathrm{DC}$ are more susceptible to PA induced cell death compared with epithelial cells and that at least part of the observed cell death is due to apoptosis.

To evaluate whether the observed results for A549 cells also hold true for other primary cultures of human lung epithelial cells, human bronchial epithelial cells and A549 cells were infected with PAO1. At 10 and $100 \mathrm{cfu} / \mathrm{cell}$, doses that cause more than $70 \%$ cell death in AM or DC, there was less than $10 \%$ cell death in the A549 cells and less than $18 \%$ in the HBEC (not shown).

Suppression of $P A$ induced $D C$ cell death by caspase inhibition. To evaluate whether caspase activation is part of
A. Clinical isolate

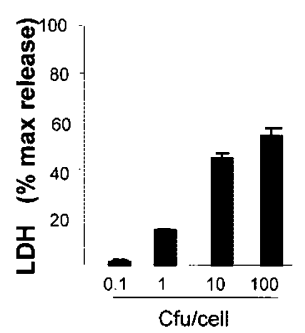

\section{Heat inactivated PA or LPS}

100

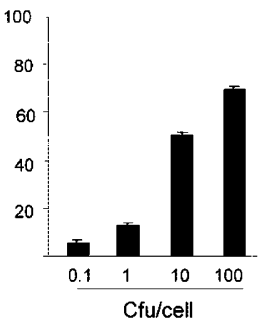

80

60

40

20

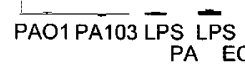

the PA induced apoptotic response of DC to PA exposure, PA induced cell death was evaluated following caspase inhibition in DC. Cell death was significantly reduced following incubation with the capase inhibitor VAD-fmk and 2VAD-cmk for exposure of DC to PAO1 ( $p<0.05$ compared with controls, Fig. 6A) and PA103 $(p<0.001$ compared with controls, Fig. $6 B)$, suggesting that caspase activation is involved in the cellular apoptotic response in the DC.

Decreased apoptosis following genetic modification of $D C$ with CD40L. DC modified with AdCD40L demonstrated expression of $\mathrm{CD} 40 \mathrm{~L}$ on the surface compared with AdNull infected or uninfected DC (Fig. 7A). The expression of MHC class II (IA-b) and CD54 (ICAM-1) did not change in AdCD40L infected cells compared with AdNull infected cells (Fig. 7B,C). To analyze whether PA induced cell death in DC was influenced by expression of CD40L, DC infected with AdCD40L were exposed to PA. DC modified with CD40L and infected with PAO1 showed 32\% and 30\% less cell death compared with naive DC or DC modified with AdNull respectively ( $p<0.001$, all comparisons, Fig. $8 A$ ). Also, DC modified with CD40L and exposed to PDO300 showed $45 \%$ and $55 \%$ less cell death compared with naive DC or DC modified with AdNull respectively ( $p<0.001$, all comparisons, Fig. $8 B$ ). Modification with AdNull or AdCD40L alone without PA exposure had no effect (not shown). The number of apoptotic DC, determined by the photometric apoptosis assay, following PAO1 infection was decreased in the cells modified with AdCD40L by $36 \%$ and $49 \%$ compared with controls or AdNull modified cells following infection with PAO1 at $1 \mathrm{cfu} / \mathrm{cell}(p<$ 0.01 all comparisons, Fig. $9 A$ ) and was decreased by $59 \%$ and $48 \%$ compared with controls or AdNull modified cells following infection with PAO1 at $10 \mathrm{cfu} / \mathrm{cell}(p<0.001$ all comparisons, Fig. 9B). The degree of cytotoxicity (Fig. 8) was higher than the amount of apoptosis observed (Fig. 9). Although these experiments were done independently, this may be a reflection of apoptosis and necrosis occurring simultaneously. Evaluating the number of apoptotic cells by TUNEL assay showed, compared with uninfected cells (Fig. $10 A-C$ ), an increased number of apoptotic cells in the AdNull or non-Ad modified controls following infection with PAO1 (Fig. 10D,E) or PDO300 (Fig. $10 G, H)$ compared with AdCD40L-modified cell (Fig. 10F, I). Quantification of the cells showing apoptotic nuclei demonstrated a decrease of $57 \%$ and $55 \%$ compared with controls or AdNull modified cells for PAO1 $(p<0.001$ compared with both controls, Fig. 11A) and a decrease of $72 \%$ and $68 \%$ compared with controls or AdNull modified cells for PDO300 ( $p<0.001$ compared with both controls, Fig. 11B). The overall number of apoptotic cells was lower than in the experiment described in Fig. 4. This, in addition to the 50\% lower dose, may be a reflection of the biologic variability of bacteria grown for different experiments. This data demonstrates that PA induced cell death in DC can be at least partially decreased using prior genetic modification to express CD40L.

\section{DISCUSSION}

The present study demonstrates that primary cultures of antigen presenting cells are more sensitive to PA induced representative of three experiments. Each datapoint represents mean of triplicate wells. 

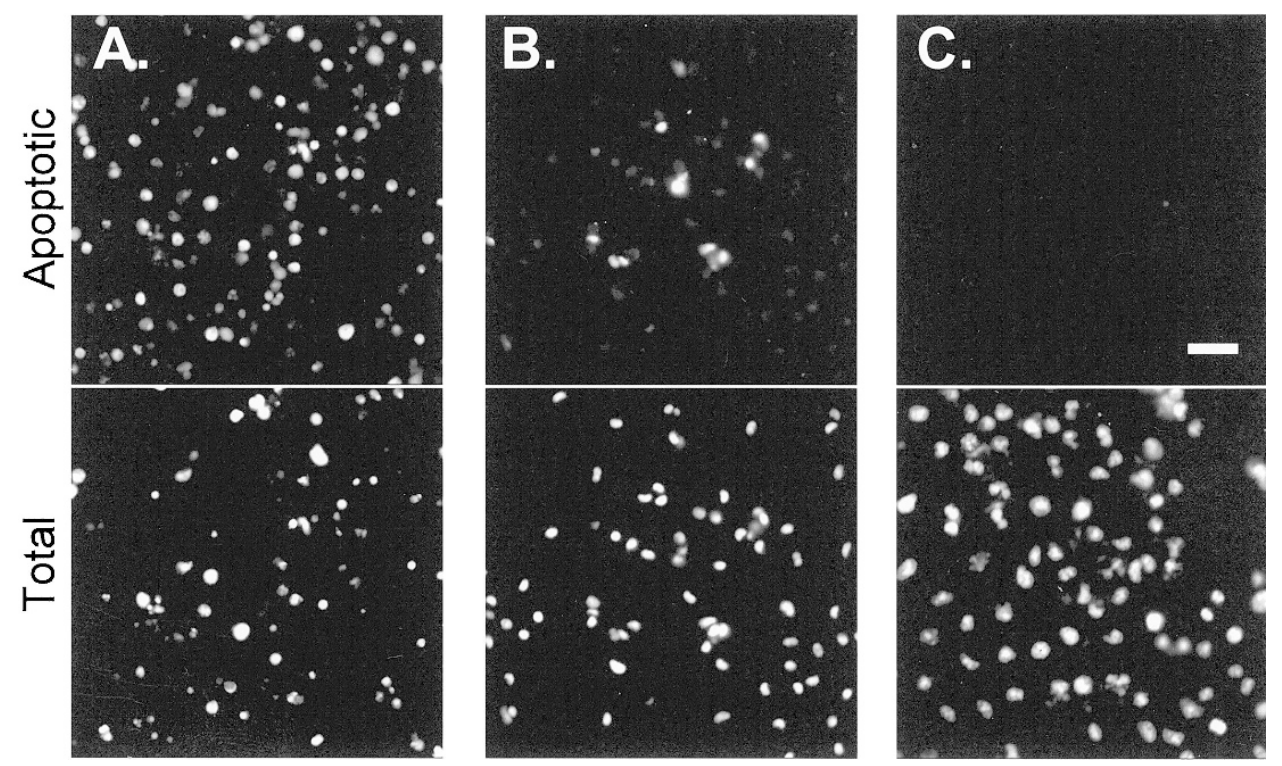

Figure 3. Pseudomonas aeruginosa induction of apoptosis in murine antigen presenting cells. Murine DC, AM and A549 cells were infected with the laboratory strain PAO1 at a dose of $10 \mathrm{cfu} / \mathrm{cell}$ for $3 \mathrm{~h}$ in coverslip dishes. $(A) \mathrm{DC} ;(B) \mathrm{AM}$, and (C) A549 cells. Apoptosis was assessed by fluorescent terminal nucleotide end labeling (TUNEL) and analyzed by fluorescent microscopy. Positive controls were generated by DNAse treatment of uninfected cells. Bar $=50 \mu \mathrm{m}$, all panels.

apoptosis than primary cultures of lung epithelial cells. The apoptotic response of the antigen presenting cells to PA was dependent on live bacteria as no cell death was observed at identical doses with heat inactivated bacteria nor with LPS at a dose approximately $10 \times$ higher than the LPS present on the bacteria, and was equally observed with a clinical isolate and a mucoid derivative. Inhibition of caspases reduced the degree of cell death. Importantly, genetic modification of DC to express CD40L diminished the cell death induced by PA. Together, these observations support the concept that PA may suppress host defenses, in part, by inducing cytotoxicity of the cells that encounter the pathogen and present it to the host mechanisms that help suppress the infection. The role of APC in the pathogenesis of $\mathrm{CF}$ is not clear, however, the immune system is not able to clear PA from the lung efficiently. Opsonic phagocytosis may be defective in the lungs of $\mathrm{CF}$ patients chronically colonized with PA, as host and bacterial proteases cleave complement and phagocytosis receptors (34). This could lead to deficient antigen presentation. In this context, the elimination of antigen presenting cells via apoptosis could lead to impairment of innate immune functions and could thus be a factor in the establishment of chronic colonization with PA. Importantly, genetic modification of the antigen presenting cells may function to prevent this action of the PA.

Induction of apoptosis by $\boldsymbol{P A}$. Various bacterial pathogens, especially Gram-negative organisms such as Salmonella, Shigella and E.coli, have the ability to induce apoptosis in host cells via a variety of bacterial factors (17-22). Recently it has become evident that PA can also induce apoptosis in host cells, so that this mechanism may be an important factor for the pathogenicity of this organism (10-16). As the lung is one of the primary sites for infection with PA, especially for patients with cystic fibrosis, most studies have focused on cells related to this organ. Studies analyzing bacterial factors responsible for the induction of apoptosis have focused on the role of the type III secretion system, although other bacterial factors have also been shown to play a role $(10,11,13,16)$. Generally, type III dependent factors can induce apoptosis or cytoskeletal changes in macrophages. For example, type III mediated induction of apoptosis in intestinal macrophages by Shigella or Yersinia leading to the release of proinflammatory cytokines is an important characteristic of the host pathogen interaction for these bacteria $(17-19,21,22)$. The type III secretion system is highly active in PA strains such as PA103 and PAO1, the strains that induced cell death most potently in the antigen presenting cells in the present study. PA103 induced apoptosis has been reported for the murine macrophage cell line J774, bone marrow derived murine macrophages and human peripheral monocytes (13). Immunization against PcrV, a component of the type III secretion system, prevents PA103 mediated cytotoxicity for AM in mice in vivo leading to increased survival and decreased pulmonary toxicity (35). Another study reported type III secretion system dependent swelling of cells and nuclei with disintegration of the plasma membrane in human leukocytes and $\mathrm{J} 774$ cells in response to PA isolates derived from individuals with $\mathrm{CF}$ (36). Nucleoside diphosphate kinase secreted by a mucoid strain of PA has been reported as another virulence factor in macrophages, inducing ATPdependent cytotoxic activity mediated through $\mathrm{P} 2 \mathrm{Z}$ receptors $(16,37)$.

The exact trigger for the cell death of the antigen presenting cells in our study is not known. Apoptosis and necrosis are visible following exposure to PA. This may be due to secondary necrosis following apoptosis, but may also reflect a direct cytotoxic effect in addition to apoptosis. Based on the variety of factors produced by the bacteria under our growth condition the latter is more likely. Comparing cytotoxicity as determined by LDH release with the number of apoptotic cells could 


\section{A. Dendritic cells}
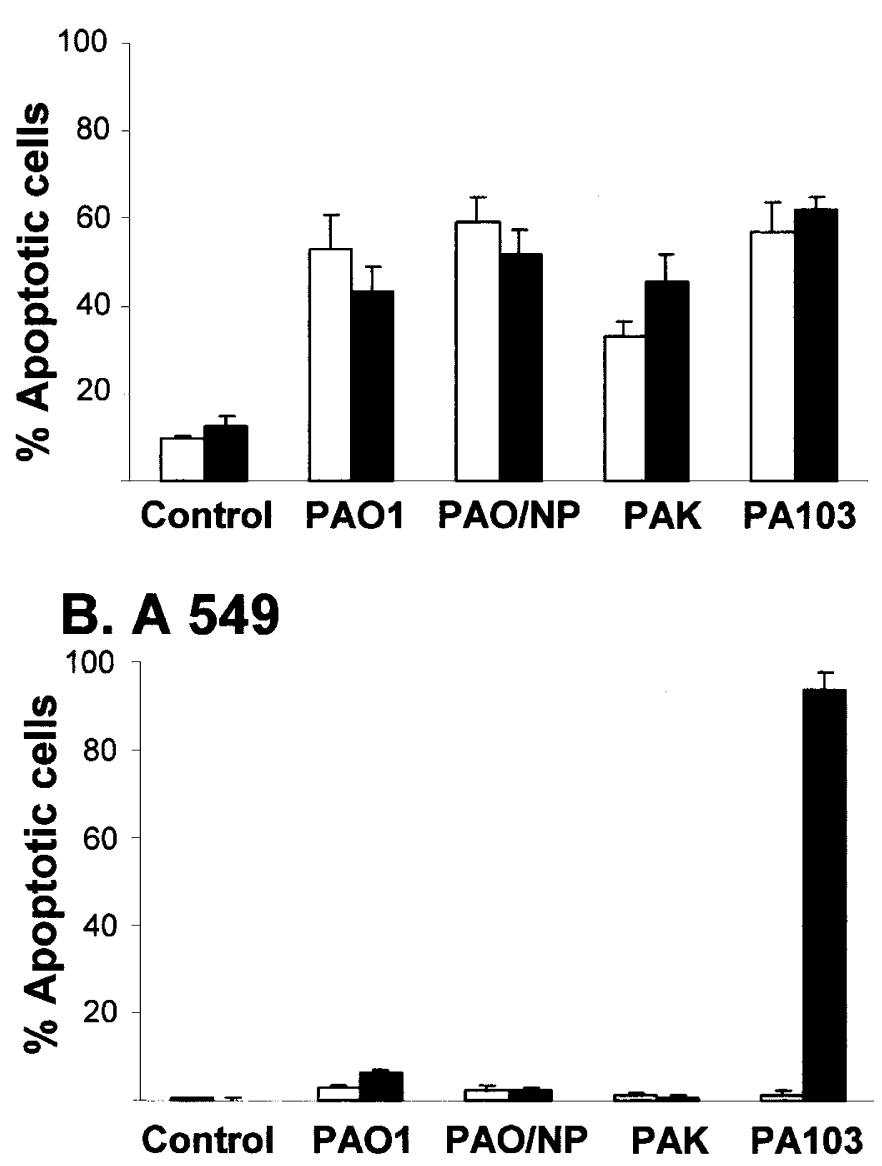

Figure 4. Quantification of apoptotic DC following exposure to Pseudomonas aeruginosa as assessed by TUNEL assay. Murine DC and A549 epithelial cells were infected with various laboratory strains of PA at a dose of $10 \mathrm{cfu} / \mathrm{cell}$ for $3 \mathrm{~h}(\square)$ or $6 \mathrm{~h}(\square)$ in coverslip dishes. Apoptosis was assessed by fluorescent terminal nucleotide end labeling (TUNEL) and analyzed by fluorescent microscopy (see Fig. 3) by counting the percentage of apoptotic cells in 5 high power fields.

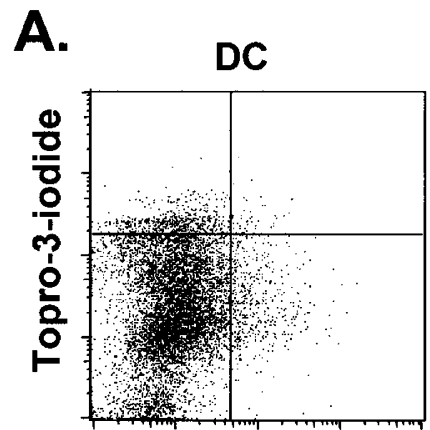

Annexin-FITC

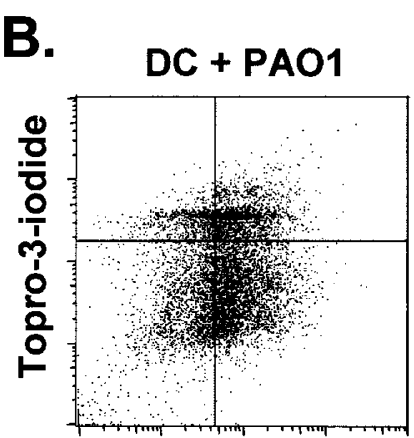

Annexin-FITC
Figure 5. Evaluation of apoptosis in DC following exposure to PA using binding of annexin V. DC were infected with PAO1 (10 cfu) for $3 \mathrm{~h} .(A)$ uninfected DC; $(B)$ DC + PAO1. The cells were stained with annexin V-FITC and topro-3 iodide and analyzed by flow cytometry. There is increased annexin staining in the DC exposed to PAO1 indicating apoptosis. Shown is a representative of four experiments.

suggest that if the apoptosis percentages are low, the mechanism of action of $P$. aeruginoda is probably a direct effect on cell permeability and if the apoptosis and cytotoxicity percent-
A. PAO1

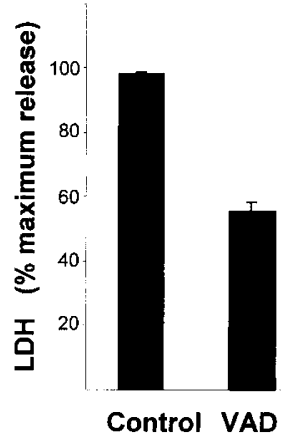

B. PA103

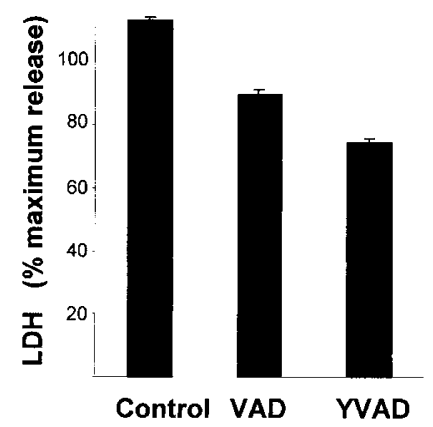

Figure 6. Induction of cell death by Pseudomonas aeruginosa in murine DC is partially suppressed by caspase inhibition. DC were infected with PA01 (10 cfu) or PA103 (1 cfu) in the presence of the caspase inhibitors VAD-fmk or YVAD-cmk. Cytotoxicity was assessed by measuring LDH release into the supernatant using a colorimetric assay following $3 \mathrm{~h}$ infection. Data are presented as LDH in supernatant/maximum cellular release of LDH by lysis with triton-X above baseline LDH release for uninfected cells. Shown is a representative of three experiments. Each datapoint represents mean of triplicate wells.
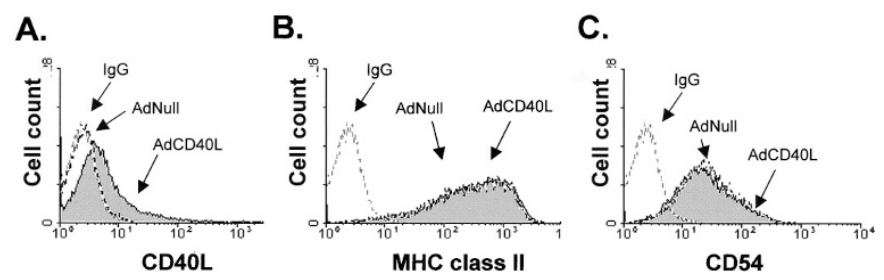

Figure 7. Expression of CD40L on DC genetically modified to express CD40L. DC were infected with AdCD40L for $24 \mathrm{~h}$ and analyzed for the expression of $(A)$ CD40L, $(B)$ MHC class II, and (C) ICAM (CD54) using PE-conjugated antibodies by flow cytometry. Shown in each panel is the result for the AdCD40L and AdNull infected cells as well as Ig-control on AdCD40L infected cells.
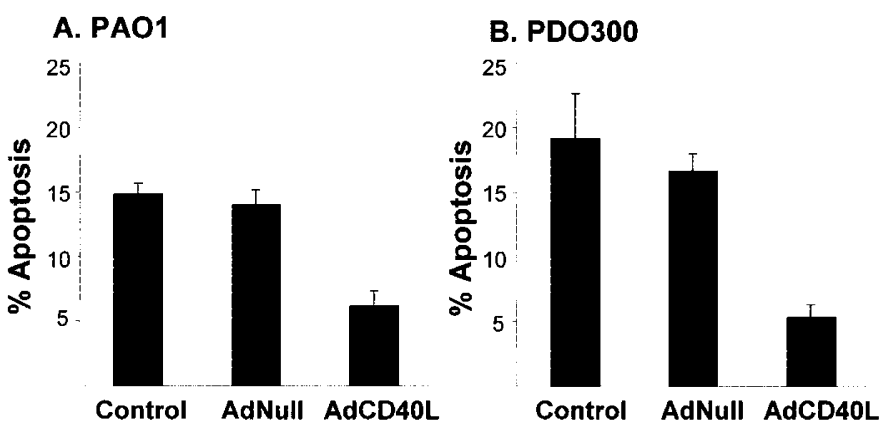

Figure 8. DC modified to express CD40L are less susceptible to cytotoxicity induced by Pseudomonas aeruginosa. DC infected with AdCD40L (100 moi) and $24 \mathrm{~h}$ later infected with PAO1 or PDO300 at $1 \mathrm{cfu} / \mathrm{cell}$. AdNull infected or uninfected DC served as controls. Cytotoxicity was assessed by measuring LDH release into the supernatant using a colorimetric assay following $3 \mathrm{~h}$ infection. Data are presented as LDH in supernatant/maximum cellular release of LDH by lysis with triton-X above baseline LDH release for uninfected cells. $(A)$ DC infected with PAO1, and $(B)$ DC infected with PDO300. Each datapoint represents mean of triplicate measurements.

ages are high and comparable, the main target of the bacteria could be the apoptotic pathway followed by secondary necrosis. The most potent strains in inducing cell death were PAO1 and PA103, both of which express the type III secretion system as a pathogenetic factor with only PA103 expressing the type 


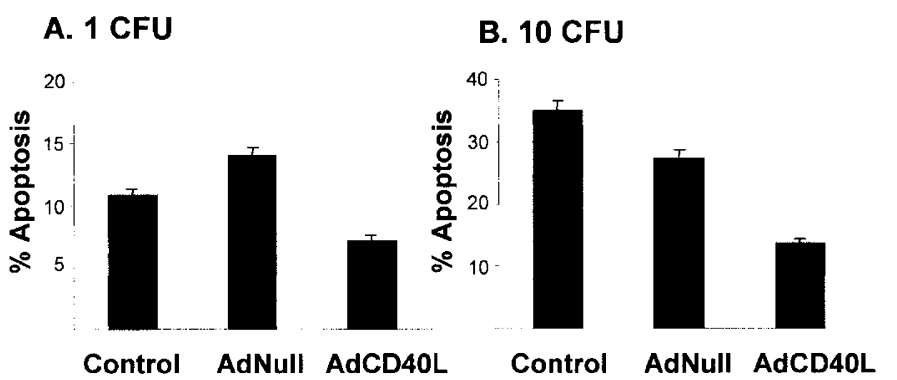

Figure 9. Inhibition of Pseudomonas aeruginosa-induced apoptosis in DC modified to express CD40L. DC genetically modified with AdCD40L (100 moi) were infected $24 \mathrm{~h}$ later with PAO1. AdNull infected DC served as control. Apoptosis was assessed by colorimetric detection of DNA fragmentation. (A) DC infected with $1 \mathrm{cfu} / \mathrm{cell} ;(B)$ DC infected with $10 \mathrm{cfu} / \mathrm{cell}$. Data are presented as \% apoptosis above baseline (cells cultured with PA for $0 \mathrm{~h}$ ) of DNAse treated cells. Each datapoint represents the mean \pm SEM of triplicate wells.

III cytotoxin exoU. The apoptotic responses did not differ significantly. Adhesion of the bacteria via pili may be a factor, as $\mathrm{PAO} / \mathrm{NP}$, a strain that lacks pilin, was less potent at similar doses, but was still able to induce cell death of the alveolar macrophages and DC. The complex rich medium used for all of our experiments may not permit the identification of PAspecific factors for the induction of apoptosis, but on the other hand enabled the comparisons of various strains under optimal growth conditions. Using more defined (e.g. iron-depleted) media instead of complex medium, may more closely reflect the growth condition in the CF lung (38) and will be analyzed in future experiments, together with the effects of epithelial lining fluid derived from patients with CF. Differences were also seen in the response to isolates generated from sputum of individuals with $\mathrm{CF}$, although no clear pattern was detectable for early or late isolates. One characteristic feature of the PA strains found in the $\mathrm{CF}$ lung is their mucoid character. $\mathrm{Mu}-$ coidity of isolates propagated in vitro is often lost when grown under standard conditions. The laboratory mucoid mutant used in our study, PDO300 was equally able to induce cell death in DC compared with its nonmucoid parental strain PAO1. The laboratory mucoid phenotype may not however reflect the complex forms of mucoidity and biofilms in the CF airways.

Host cell specific factors related to the induction of PA induced cell death have also been studied. For example, respiratory epithelial cells have been reported to be less susceptible than other mucosal epithelium to PA cytotoxicity and apoptosis
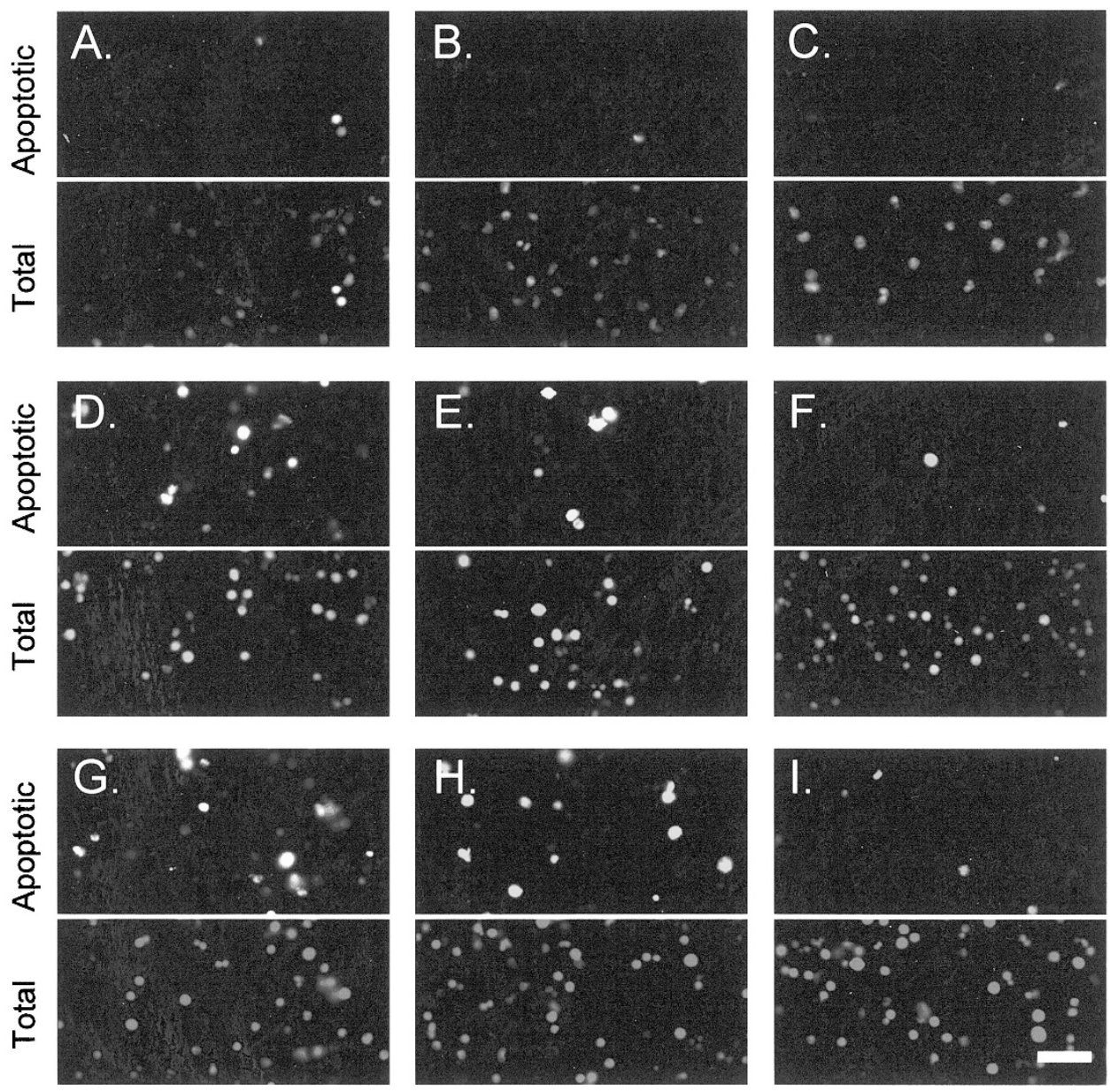

Figure 10. Decreased Pseudomonas aeruginosa-induced apoptosis in DC modified to express CD40L. Murine DC genetically modified with AdCD40L (100 moi) were infected $24 \mathrm{~h}$ later with PAO1 or PDO300 in coverslip dishes. AdNull infected DC or uninfected DC served as control. (A) Control cells uninfected, $(B)$ AdNull uninfected, $(C)$ AdCD40L uninfected, $(D)$ control cells + PAO1, $(E)$ AdNull + PAO1, $(F)$ AdCD40L + PAO1, $(G)$ control cells + PDO300, $(H)$ AdNull + PDO300, and $(I)$ AdCD40L + PDO300. Apoptosis was assessed by fluorescent terminal nucleotide end labeling (TUNEL) and analyzed by fluorescent microscopy. Bar $=50 \mu \mathrm{m}$, all panels. 


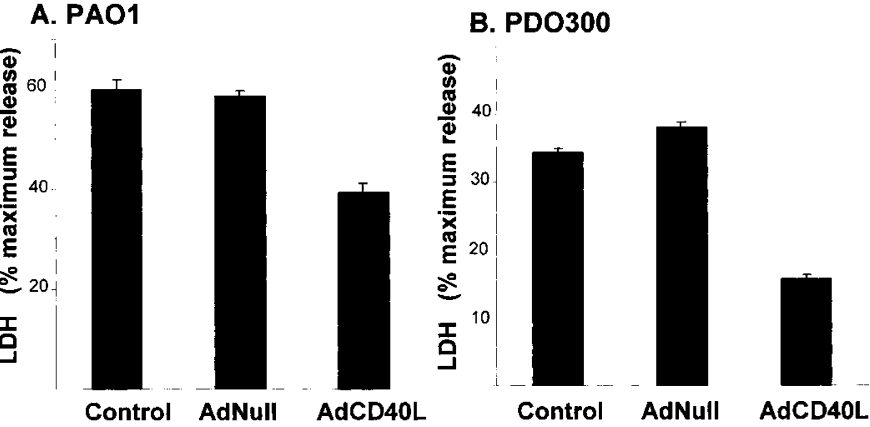

Figure 11. Quantification of apoptotic DC genetically modified to express CD40L following exposure to Pseudomonas aeruginosa as assessed by TUNEL assay. Murine DC were infected with the laboratory strains PAO1 and PDO300 at a dose of $5 \mathrm{cfu} / \mathrm{cell}$ for $3 \mathrm{~h}$ in coverslip dishes. Apoptosis was assessed by fluorescent terminal nucleotide end labeling (TUNEL) and analyzed by fluorescent microscopy by counting the percentage of apoptotic cells in 5 high power fields.

in vitro and in vivo $(13,14)$, possibly due to inaccessibility of the PA to the basolateral surface of the epithelia. The lung epithelial cells tested in the present study were less susceptible to PA induced cell death compared with antigen presenting cells under conditions without tight junctions. As the lung is an important site of infection with PA, especially in individuals with $\mathrm{CF}$, recent studies have examined induction of apoptosis in cells of the respiratory tract in vitro and in vivo. Induction of apoptosis in lung epithelial cells by various PA strains was shown in CF and non-CF cell lines and was independent of the expression of the cystic fibrosis transmembrane conductance regulator gene, the gene responsible for CF (14).

Caspase activation, a central pathway in the cellular responses to apoptotic stimuli (39), was likely involved in the cell death induced by PA. Prior treatment with the caspase inhibitors VAD-fmk and YVAD-cmk lead to decreased cell death induced by PA. These inhibitors, are rather nonspecific and do not allow identification of the individual caspases involved, however, they demonstrate that apoptosis via the caspase pathway is occurring. Fas/FasL interaction has recently been shown to be important for apoptosis induced by PA in the respiratory tract of mice in vivo (12). This pathway may play a role in the cell death induced in the antigen presenting cells, although murine DC have been shown to be resistant to apoptosis mediated via this pathway (40). Previous studies from our laboratory have demonstrated that DC pulsed with live or heat inactivated PA can induce protective immunity against pulmonary PA infection in a mouse model. The protective effect seemed equal for the heat inactivated as for the live bacteria, although we observed more cell death and less in vitro IL-12 production following preparation of the DC incubated with live bacteria (41). Apoptosis of the DC could have had an effect and influenced the immune response.

CD40L and the prevention of apoptosis. As CD40L has been shown to regulate apoptosis in B cells (24), genetic manipulation using CD40L may be a way to circumvent PA induced cell death. CD40L, a member of the tumor necrosis factor gene superfamily, is usually expressed on activated lymphocytes and functions mainly via its interaction with
$\mathrm{CD} 40$ as a mediator of $\mathrm{T}$ cell dependent $\mathrm{B}$ cell activation, proliferation and differentiation (23). Retrovirus-mediated expression of CD40L in tumor cells inhibited tumor-induced apoptosis following ligation to CD40 (25). Genetic modification of DC to express CD40L before infection with PA significantly reduced the amount of PA induced cell death for the nonmucoid strain PAO1 as well as the mucoid strain PDO300. AdNull infection in itself seemed to have a small effect on apoptosis. Although the differences were statistically not significant and not consistent, Ad infection may influence cell survival independent of the transgene expression. Concomitant expression of CD40L with antigens in DC has been shown to activate $\mathrm{DC}$ and induce antibody production by $\mathrm{B}$ cells independent of $\mathrm{CD} 4+$ cells, although the mechanism of how gene transfer of $\mathrm{CD} 40 \mathrm{~L}$ to $\mathrm{DC}$ activates the $\mathrm{DC}$ has not been elucidated (26). Expression of CD40L acting within the cells, or expression of the protein on the surface leading to activation through interaction with CD40 on the same or neighboring cells are potential mechanisms. Surface expression of CD40L in the present study was detectable for about $50 \%$ of the cells, although the number of CD40L transduced cells may be higher, as CD40L can be shed from the surface (23). Whether or not the expression of CD40 induced by gene transfer of $\mathrm{CD} 40 \mathrm{~L}$ is necessary is not known, as all the antigen presenting cells used in the present study expressed CD40 on their surface. Antibodies against CD40 to block this receptor usually lead to activation and thus may not answer the question. Genetic modification of cell to resist PA-induced apoptosis could be used to improve host defenses against PA. The concept that expression of $\mathrm{CD} 40 \mathrm{~L}$ in antigen presenting cells will prevent cell death induced by bacterial pathogens is a novel concept that warrants further investigation.

Acknowledgments. The authors thank W. van't Hof, N.R. Hackett, and P.L. Leopold, for helpful discussions; and N. Mohamed for help in preparing this manuscript.

\section{REFERENCES}

1. Davis PB, Drumm M, Konstan MW 1996 Cystic fibrosis. Am J Respir Crit Care Med 154:1229-1256

2. Govan JR Deretic V 1996 Microbial pathogenesis in cystic fibrosis: mucoid Pseudomonas aeruginosa and Burkholderia cepacia. Microbiol Rev 60:539-574

3. Tummler B Kiewitz C 1999 Cystic fibrosis: an inherited susceptibility to bacterial respiratory infections. Mol Med Today 5:351-358

4. Davies DG, Parsek MR, Pearson JP, Iglewski BH, Costerton JW, Greenberg EP 1998 The involvement of cell-to-cell signals in the development of a bacterial biofilm. Science 280:295-298

5. Singh PK, Schaefer AL, Parsek MR, Moninger TO, Welsh MJ, Greenberg EP 2000 Quorum-sensing signals indicate that cystic fibrosis lungs are infected with bacterial biofilms. Nature 407:762-764

6. Bezdicek P, Crystal RG 1997 Pulmonary macrophages. In: Crystal RG, West JB, Weibel ER, P.J. Barnes (eds) The Lung: Scientific Foundation. Lippincott-Raven Inc., Philadelphia, pp 859-875

7. McWilliam AS, Nelson DJ, Holt PG 1995 The biology of airway dendritic cells. Immunol Cell Biol 73:405-413

8. McWilliam AS, Stumbles PA, Holt PG. Dendritic Cells in the Lung 1999 In: Lopez MT, Thomson AW (eds) Dendritic Cells: Biology and Clinical Applications. Academic Press, San Diego, pp 123-140

9. Reynolds HY 1997 Integrated Host Defenses Against Infection. In: Crystal RG, West JB, Weibel RE, Barnes PJ (eds) The Lung: Scientific Foundations. Lippincott-Raven, Inc., Philadelphia, pp 2353-2366

10. Bruno TF, Woods DE, Mody CH 2000 Exoenzyme S from Pseudomonas aeruginosa induces apoptosis in T lymphocytes. J Leukoc Biol 67:808-816

11. Coburn J Frank DW 1999 Macrophages and epithelial cells respond differently to the Pseudomonas aeruginosa type III secretion system. Infect Immun 67:3151-3154 
12. Grassme H, Kirschnek S, Riethmueller J, Riehle A, von Kurthy G, Lang F, Weller M, Gulbins E 2000 CD95/CD95 ligand interactions on epithelial cells in host defense to Pseudomonas aeruginosa. Science 290:527-530

13. Hauser AR Engel JN 1999 Pseudomonas aeruginosa induces type-III-secretionmediated apoptosis of macrophages and epithelial cells. Infect Immun 67:5530-5537

14. Rajan S, Cacalano G, Bryan R, Ratner AJ, Sontich CU, van Heerckeren A, Davis P, Prince A 2000 Pseudomonas aeruginosa induction of apoptosis in respiratory epithelial cells: analysis of the effects of cystic fibrosis transmembrane conductance regulator dysfunction and bacterial virulence factors. Am J Respir Cell Mol Biol 23:304-312

15. Valente E, Assis MC, Alvim IM, Pereira GM, Plotkowski MC 2000 Pseudomonas aeruginosa induces apoptosis in human endothelial cells. Microb Pathog 29:345-356

16. Zaborina O, Dhiman N, Ling CM, Kostal J, Holder IA, Chakrabarty AM 2000 Secreted products of a nonmucoid Pseudomonas aeruginosa strain induce two modes of macrophage killing: external-ATP-dependent, P2Z-receptor-mediated necrosis and ATP-independent, caspase-mediated apoptosis. Microbiology 146:2521-2530

17. Donnenberg MS 2000 Pathogenic strategies of enteric bacteria. Nature 406:768-774

18. Gao LY Kwaik YA 2000 The modulation of host cell apoptosis by intracellular bacterial pathogens. Trends Microbiol 8:306-313

19. Hersh D, Monack DM, Smith MR, Ghori N, Falkow S, Zychlinsky A 1999 The Salmonella invasin SipB induces macrophage apoptosis by binding to caspase-1. Proc Natl Acad Sci USA 96:2396-2401

20. Monack DM, Mecsas J, Bouley D, Falkow S 1998 Yersinia-induced apoptosis in vivo aids in the establishment of a systemic infection of mice. J Exp Med 188:2127-2137

21. Yrlid U Wick MJ 2000 Salmonella-induced apoptosis of infected macrophages result in presentation of a bacteria-encoded antigen after uptake by bystander dendritic cells. J Exp Med 191:613-624

22. Zychlinsky A Sansonetti PJ 1997 Apoptosis as a proinflammatory event: what can we learn from bacteria-induced cell death? Trends Microbiol 5:201-204

23. Grewal IS Flavell RA 1998 CD40 and CD154 in cell-mediated immunity. Annu Rev Immunol 16:111-135

24. Kehry MR 1996 CD40-mediated signaling in B cells. Balancing cell survival, growth, and death. J Immunol 156:2345-2348

25. Esche C, Gambotto A, Satoh Y, Gerein V, Robbins PD, Watkins SC, Lotze MT, Shurin MR 1999 CD154 inhibits tumor-induced apoptosis in dendritic cells and tumor growth. Eur J Immunol 29:2148-2155

26. Kikuchi T, Worgall S, Singh R, Moore MA, Crystal RG 2000 Dendritic cells genetically modified to express CD40 ligand and pulsed with antigen can initiate antigen-specific humoral immunity independent of CD4 + T cells. Nat Med 6:1154-1159

27. Song W, Kong HL, Carpenter H, Torii H, Granstein R, Rafii S, Moore MA, Crysta RG 1997 Dendritic cells genetically modified with an adenovirus vector encoding the cDNA for a model antigen induce protective and therapeutic antitumor immunity. J Exp Med 186:1247-1256

28. Russi TJ, Crystal RG 1997 Bronchoalveolar Lavage. In: Crystal RG, West JB, Weibel ER, P.J. Barnes (eds) The Lung: Scientific Foundation. Lippincott-Raven Inc., Philadelphia, pp 371-382
29. Kikuchi T Crystal RG 1999 Anti-tumor immunity induced by in vivo adenovirus vector-mediated expression of CD40 ligand in tumor cells. Hum Gene Ther 10:13751387

30. Hersh J, Crystal RG, Bewig B 1995 Modulation of gene expression after replicationdeficient, recombinant adenovirus-mediated gene transfer by the product of a second adenovirus vector. Gene Ther 2:124-131

31. Rosenfeld MA, Siegfried W, Yoshimura K, Yoneyama K, Fukayama M, Stier LE, Paakko PK, Gilardi P, Stratford-Perricaudet LD, Perricaudet M, Jallat S, Pavirani A, Lecocq J-P, Crystal RG 1991 Adenovirus-mediated transfer of a recombinant alpha 1-antitrypsin gene to the lung epithelium in vivo. Science 252:431-434

32. Rosenfeld MA, Yoshimura K, Trapnell BC, Yoneyama K, Rosenthal ER, Dalemans W, Fukayama M, Bargon J, Stier LE, Stratford-Perricaudet L, Perricaudet M, Guggino WB, Pavirani A, Lecocq J-P, Crystal RG 1992 In vivo transfer of the human cystic fibrosis transmembrane conductance regulator gene to the airway epithelium. Cell 68:143-155

33. Crystal RG, McElvaney NG, Rosenfeld MA, Chu CS, Mastrangeli A, Hay JG, Brody SL, Jaffe HA, Eissa NT, Danel C 1994 Administration of an adenovirus containing the human CFTR cDNA to the respiratory tract of individuals with cystic fibrosis. Nat Genet 8:42-51

34. Tosi MF, Zakem H, Berger M 1990 Neutrophil elastase cleaves C3bi on opsonized pseudomonas as well as CR1 on neutrophils to create a functionally important opsonin receptor mismatch. J Clin Invest 86:300-308

35. Sawa T, Yahr TL, Ohara M, Kurahashi K, Gropper MA, Wiener-Kronish JP, Frank DW 1999 Active and passive immunization with the Pseudomonas V antigen protects against type III intoxication and lung injury. Nat Med 5:392-398

36. Dacheux D, Toussaint B, Richard M, Brochier G, Croize J, Attree I 2000 Pseudomonas aeruginosa cystic fibrosis isolates induce rapid, type III secretion-dependent, but ExoU-independent, oncosis of macrophages and polymorphonuclear neutrophils. Infect Immun 68:2916-2924

37. Zaborina O, Misra N, Kostal J, Kamath S, Kapatral V, El-Idrissi ME, Prabhakar BS, Chakrabarty AM 1999 P2Z-Independent and P2Z receptor-mediated macrophage killing by Pseudomonas aeruginosa isolated from cystic fibrosis patients. Infect Immun 67:5231-5242

38. Sawa T, Ohara M, Kurahashi K, Twining SS, Frank DW, Doroques DB, Long T, Gropper MA, Wiener-Kronish JP 1998 In vitro cellular toxicity predicts Pseudomonas aeruginosa virulence in lung infections. Infect Immun 66:3242-3249

39. Earnshaw WC, Martins LM, Kaufmann SH 1999 Mammalian caspases: structure, activation, substrates, and functions during apoptosis. Annu Rev Biochem 68:383424

40. Ashany D, Savir A, Bhardwaj N, Elkon KB 1999 Dendritic cells are resistant to apoptosis through the Fas (CD95/APO-1) pathway. J Immunol 163:5303-5311

41. Worgall S, Kikuchi T, Singh R, Martushova K, Lande L, Crystal RG 2001 Protection against pulmonary infection with Pseudomonas aeruginosa following immunization with P. aeruginosa-pulsed dendritic cells. Infect Immun 69:4521-4527 